\title{
Prevalence and distribution of colonic diverticulosis in patients undergoing colonoscopy. Four years' experience from a single center in Warsaw
}

\author{
Krzysztof A. Hadrian ${ }^{1}$, Anna Basaj ${ }^{2}$, Piotr Nehring ${ }^{1}$, Bartłomiej A. Ziółkowski ${ }^{1}$
}

\author{
${ }^{1}$ Department of Gastroenterology and Internal Medicine, Medical University \\ of Warsaw, Warsaw, Poland \\ ${ }^{2}$ Trinity College, University of Cambridge, Cambridge, United Kingdom
}

Submitted: 21 March 2016

Accepted: 24 April 2016

Arch Med Sci Civil Dis 2016; 1: e30-e35

DOI: 10.5114 /amscd.2016.60164

Copyright @ 2016 Termedia \& Banach

\section{Abstract}

Introduction: Data on the prevalence of diverticulosis in the Polish population are limited. Most patients are asymptomatic, so it is difficult to estimate the scale of the problem in the population. The aim of this study was to determine the prevalence of colonic diverticulosis in Polish adults based on a sample from a single, medium-sized endoscopy department. The secondary objective was to assess the distribution of diverticula in the colon and their association with sex and age.

Material and methods: Between 2010 and 2014 a total of 6087 consecutive patients (age range: 18-94 years, mean age: 54 years) underwent colonoscopy in the department. 1981 patients were excluded from the study due to incomplete examination, colorectal surgery or inadequate preparation resulting in insufficient visibility. The remaining 4106 patients were included in the study (1648 - male, 2458 - female). Data concerning age, sex, and presence and location of diverticula were studied in a retrospective manner. Results: Diverticula were found in $29.6 \%$ of all participants $(29.4 \%$ - male, $29.8 \%$ - female). $0.5 \%$ of patients $(0.7 \%$ - male, $0.5 \%$ - female) had right-sided diverticulosis, while in $23.7 \%$ (23.4\% - male, $24.0 \%$ - female) diverticula were located in the left colon only. Pancolonic diverticulosis was present in $4.0 \%$ (4.0\% - male, 4.0\% - female). For all statistical calculations $p \leq 0.05$. The prevalence of diverticulosis increased with age. No difference was noted between men and women.

Conclusions: In the studied sample, diverticula predominantly occurred in the sigmoid and descending colon. Their prevalence and distribution were similar to other Caucasian populations.

Key words: colon, diverticulum, prevalence.

\section{Introduction}

The presence of diverticula in the colon, known widely as colonic diverticulosis, is an important worldwide problem, which has an increasing prevalence in developed countries [1].

By definition, in acquired left-sided diverticulosis, diverticula represent usually multiple herniations of the colonic mucosa and sub-mucosa, also called pseudodiverticula. True diverticula, involving all 3 layers of the colonic wall, are typical of congenital and right-sided variants of the disease, relatively rare in Western countries [2].

\author{
Corresponding author: \\ Bartłomiej Ziółkowski \\ $\mathrm{MD}, \mathrm{PhD}$ \\ Department of \\ Gastroenterology \\ and Internal Medicine \\ Medical University of Warsaw \\ 1 a Banacha St \\ 02-094 Warsaw, Poland \\ Phone: +48 225992838 \\ Fax: +48 225991838 \\ E-mail: bartlomiej. \\ ziolkowski@wum.edu.pl
}


It is important to highlight that while diverticulosis is very common, affecting up to $60 \%$ of adults at the age of 60 , diverticular disease (complicated diverticulosis) develops only in $4-20 \%$ of patients [3]. Still, diverticulitis - one of the complications of diverticulosis - is quite a common reason for emergency admission of elderly patients with non-traumatic abdominal pain [4]. Even though recent studies show that the estimated incidence of diverticulosis progressing to diverticulitis is low, the costs generated by this condition are a significant burden to health care systems [5].

The pathogenesis of diverticulosis is complex. It has been believed that low fiber intake, hard stools and constipation are responsible for diverticula formation $[6,7]$. Recent findings, however, suggest no influence of a low-fiber diet on diverticulosis. Furthermore, a number of studies have revealed that patients with less frequent, harder stools face a lower risk of diverticulosis $[8,9]$.

It is suggested that one of the contributing factors is the specific anatomical structure of the colonic wall with intrinsic weak points, which are less resistant to high intraluminal pressure [10]. Age-related increase of collagen cross-links, losing the compliance to accommodate increased intraluminal pressures and some genetic predispositions may be an issue as well [11-13].

The prevalence of diverticulosis differs among the continents. In Asia the occurrence rate is lower but still significant. Studies from Asian countries from various geographic areas show mainly proximal diverticulosis [14]. Conversely, data from Western Europe and the United States show domination of the left-sided localization. The relatively low prevalence in Africa may be attributed to short life expectancy, dietary and genetic differences or underestimation due to difficult access to medical services [15].

There are scant data on the prevalence of colonic diverticulosis in Poland. Searches in available internet databases showed that the only study investigating this area so far was a small barium enema study carried out in the last decade in the Silesia region [16].

\section{Material and methods}

We retrospectively reviewed reports of 6087 consecutive patients who underwent colonoscopy between 2/8/2010 and 21/8/2014 in the Department of Gastroenterology and General Medicine, Medical University of Warsaw, Poland. The data were collected from two databases: the endoscopy department database and the National Colorectal Cancer Screening Program database. The latter consisted of 1060 asymptomatic patients (17.4\%) aged between 40 and 65 . The overall caecum intubation rate (CIR) was $92.95 \%$.
To avoid bias, the authors decided to exclude from the study patients with a history of any colorectal surgery, which might have resulted in disturbances of anatomical relations as well as in false negative findings. Other exclusion criteria were incomplete examination and poor or inadequate preparation of any segment of the colon, resulting in insufficient visibility during the procedure, precluding correct assessment of the examined organ. A total of 1981 (32.6\%) patients were excluded based on the above criteria. The remaining 4106 were included in the study (1648 - male, 2458 - female).

Information from the electronic records included age at the date of examination, sex, completion of the procedure, adequacy of preparation, findings and the performing physician's identity. Diverticulosis was defined as the presence of one or more saccular herniations inside the wall of the colon. The location was defined in two ways: primary as left-sided (including the sigmoid and descending colon), middle (including the transverse colon with both flexures) and right-sided (including the caecum and ascending colon). For the purposes of this study and for clear presentation, it was necessary to introduce five separate anatomical regions (caecum, ascending colon, transverse colon with both flexures, descending colon, and sigmoid colon).

\section{Statistical analysis}

Statistical analysis was done using a Student's $t$-test modified for binominal distribution with $p=$ 0.05 and the $\chi^{2}$ test, both in Microsoft Excel.

\section{Results}

Diverticula were described in 1215 (29.6\%) participants, 732 (29.8\%) females and 483 (29.3\%) males. Mean age of patients with colonic diverticulosis was 67.4 years, age range: $31-94$. Mean age of male subjects was 66.9 years and of female subjects was 67.8 years. Distribution of diverticula in the colon is shown in Table I.

No diverticula were observed in all 216 patients aged 31 years or younger. The prevalence of di-

Table I. Distribution of diverticula in the colon (note: some patients had more than one localization)

\begin{tabular}{|lcccc|}
\hline Diverticular & \multicolumn{2}{c}{ Female $(N=2458)$} & \multicolumn{2}{c|}{ Male $(N=1648)$} \\
\cline { 2 - 5 } location & $n$ & $\%$ & $n$ & $\%$ \\
\hline Sigmoid & 718 & 29.2 & 468 & 28.4 \\
\hline Descending & 174 & 7.1 & 112 & 6.8 \\
\hline Transverse & 123 & 5.0 & 73 & 4.4 \\
\hline Ascending & 121 & 4.9 & 81 & 4.9 \\
\hline Caecum & 113 & 4.6 & 63 & 3.8 \\
\hline
\end{tabular}




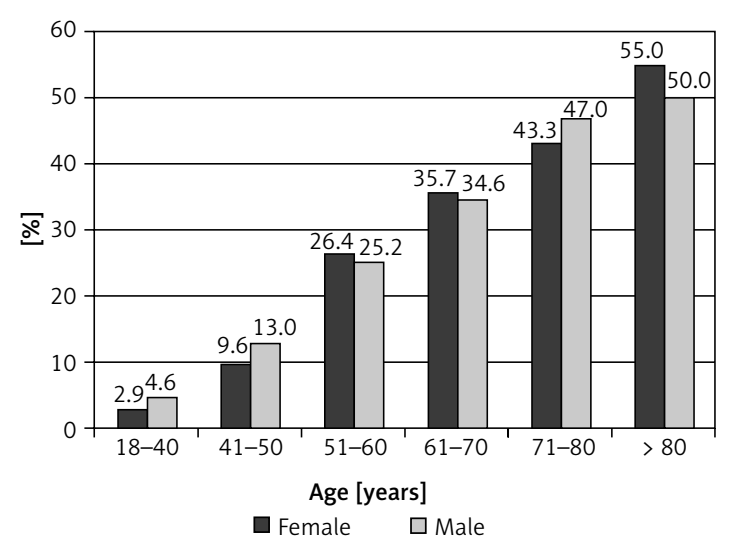

Figure 1. Prevalence of colonic diverticulosis according to gender and age

verticulosis increased with age, both in men and women (Figure 1, Table II). There were no significant differences in the prevalence of diverticula between genders.

The distribution of diverticula in the studied group was predominantly left-sided (Figure 2 ).

There were 900 (21.9\%) patients (mean age: 67.0 years, age range: $32-94$ years) with diverticula isolated in a single anatomical region; 874 of them had diverticula in the sigmoid colon. One hundred and sixty-four (4.0\%) patients had pancolonic diverticulosis (mean age: 69.3 years, age range: 40-91 years). The prevalence of diverticula in the sigmoid colon increased at the rate of $1.0 \%$ per life-year, in the descending colon at the rate of $0.3 \%$, and in other anatomical regions at the rate of $0.2 \%$ (Figure 3 ).

\section{Discussion}

This study showed high prevalence of colonic diverticulosis, similar to the prevalence reported in other Western countries. The significance of this study is an almost $8 \%$ higher prevalence of diverticulosis than described in the earlier Polish study by Blachut et al. [16]. The novelty of the

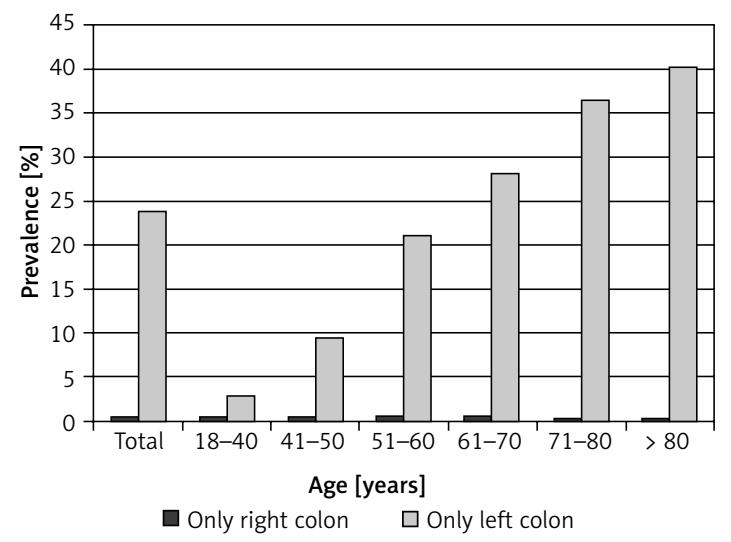

Figure 2. Distribution of diverticula in patients according to age
Table II. Prevalence of colonic diverticulosis according to gender and age

\begin{tabular}{|lccc|}
\hline $\begin{array}{l}\text { Age } \\
\text { [years] }\end{array}$ & $\begin{array}{c}\text { Total } \\
\boldsymbol{N}=1215\end{array}$ & $\begin{array}{c}\text { Female } \\
\boldsymbol{N}=732\end{array}$ & $\begin{array}{c}\text { Male } \\
\boldsymbol{N}=483\end{array}$ \\
\hline $31-40$ & 18 & $8(2.9 \%)$ & $10(4.6 \%)$ \\
\hline $41-50$ & 45 & $24(9.6 \%)$ & $21(13.0 \%)$ \\
\hline $51-60$ & 296 & $177(26.4 \%)$ & $119(25.2 \%)$ \\
\hline $61-70$ & 334 & $210(35.7 \%)$ & $124(34.6 \%)$ \\
\hline $71-80$ & 342 & $202(43.3 \%)$ & $140(47.0 \%)$ \\
\hline$>80$ & 180 & $111(55.0 \%)$ & $69(50.0 \%)$ \\
\hline
\end{tabular}

present study is mainly the inclusion of so far the largest number of patients in a prevalence study on colonic diverticulosis in Poland. Our study was based on a three times larger group, including a significant number of asymptomatic patients. The survey, carried out in Silesia, was based on barium examinations of the large bowel in symptomatic patients. In the symptomatic population, some of the symptoms may be attributed to diverticular disease, and therefore the yield of detection should actually have been higher. As in colonoscopy, detection of diverticula in a barium study can be hampered by poor preparation. Previous colonic resections can also result in some underestimation. As mentioned before, we used both these factors as exclusion criteria to avoid bias. Furthermore, collection of data during colonoscopy was not focused on diverticulosis; therefore, occasionally the endoscopist might not have recorded the presence of diverticula. Since most colonoscopies in our survey $(73.1 \%)$ were performed by a single endoscopist, we expect some underestimation due to the possibility of treating diverticula at a certain age as part of a "normal" picture in colonoscopy. We believe this single-endoscopist experience should be treated as a potential weakness of the study. Another weakness

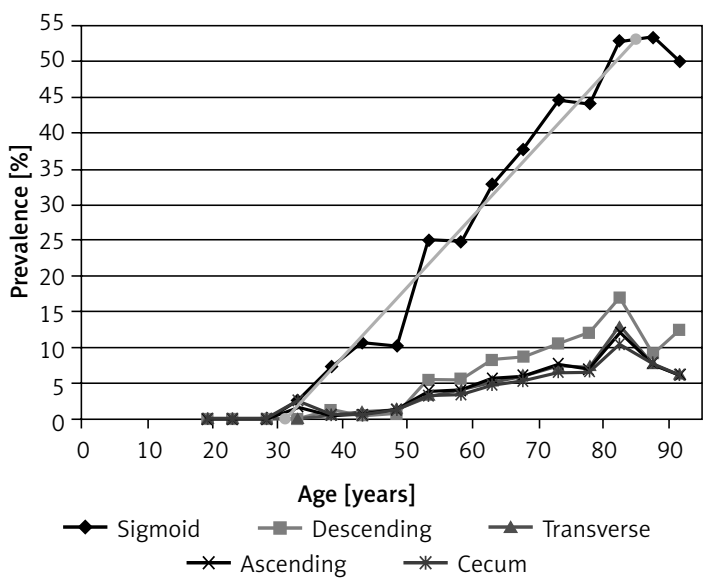

Figure 3. Distribution of diverticula in colon segments according to age 
contributing to a possible bias was the fact that only $17 \%$ of our patients were asymptomatic. In spite of these limitations, recent large epidemiological studies from Western countries have confirmed high prevalence of diverticulosis, up to $43 \%[8,17,18]$.

The closest to our results was the endoscopic survey from Holland, which reported diverticular prevalence at a very similar level of $27 \%$ [17]. A different survey, conducted in multicultural London, where participation of a large oriental minority of patients could have influenced the results, showed the prevalence of diverticulosis in the non-Indian origin population of $22 \%$ [19].

Most of the surveys, including this one, show, characteristic for industrialized Western societies, diverticular prevalence increasing with age, and predominantly with left-sided distribution.

According to some theories, highly processed food and a fiber-deficient diet are factors contributing to the development of diverticulosis. As previously mentioned, the evidence to support the role of dietary fiber intake has recently been questioned. Studies performed in this and the previous decade in different parts of the world found no evidence for associating colonic diverticulosis with a low-fiber diet $[8,14,20]$. Two Asian studies supported the theory of a positive association of diverticulosis with meat and fat intake. The theory was also supported in some of the older studies, but most of them underlined the preventive role of fiber [21, 22]. In fact, recent evidence has shown that higher fiber intake in a studied cohort resulted in increased frequency of diverticulosis [8]. Some authors would speculate that increased fiber intake in subjects with diverticulosis could be secondary to symptoms; patients would eat more fiber to prevent pain or to obtain relief [23]. Apparently, there is no strong evidence to support the beneficial role of fiber in relief of symptoms. There have only been a few randomized trials comparing fiber versus placebo in diverticular disease. Two dated, small randomized controlled trials (RCT) performed in the previous century supported the role of a high-fiber diet in improvement of symptoms [24, 25], but the results of a third RCT, twice as big, performed by Ornstein et al., gave conflicting evidence, showing improvement in constipation symptoms only, but not pain [26]. These data do not allow confirmation of the significance of a high-fiber diet in preventing the development of diverticulosis and in treating symptomatic disease. Furthermore, the study by Peery not only questioned the role of fiber, but also failed to prove any association between diverticulosis and consumption of fat, red meat, alcohol, non-steroid anti-inflammatory drugs, aspirin use, or physical activity/sedentary behavior. In view of this ev- idence, the old theory of the wrong westernized diet, causing constipation that increases intraluminal pressure and facilitates herniations of mucosa and submucosa through the weak points of intestinal smooth muscle, is called into question. The only factors that survived the unhealthy lifestyle theory are smoking and increased body mass index. Caucasian origin turned out to be a factor of diverticulosis as well, as non-Caucasian participants had a $26 \%$ lower risk after adjustment for risk factors [8].

The study by Blachut et al. showed a similar prevalence of diverticulosis in men and women, as did the results of our study. Gender does not seem to significantly influence the occurrence of diverticulosis in most of the Western papers [15, 17, 27]. Interestingly, a large retrospective study from Canada showed higher rates of hospital admissions for complicated diverticular disease in women [28], while in another study, in symptomatic but uncomplicated patients the male to female ratio was $1: 1$ [29].

In view of the new evidence negating the role of many dietary and life-style factors, it is tempting to review the genetic theory of diverticular disease. There is some evidence that genetic factors may contribute to the development of diverticulosis. Caucasian race, mentioned above, is one of the inherited factors. Many studies have shown that different ethnicities exhibit different diverticulosis prevalence rates [19]. Two recent twin studies have provided strong evidence for the heritability of diverticulosis, and it was estimated to be $40 \%$ and $53 \%[30,31]$. Some data suggest that there are genetic factors responsible for the progression of diverticulosis to diverticular disease. A small study reported a single nucleotide polymorphism apparently associated with diverticulitis requiring surgical treatment [32]. Inherited diseases of the connective tissue carry a higher risk of diverticulosis and diverticular disease. It is believed that "vulnerable" connective tissue in patients with defects in collagen synthesis causes weakness of the colonic wall, and with aging and exposure to environmental factors diverticulosis develops. Higher risk of diverticulosis and its complications apparently affects patients with Ehlers-Danlos syndrome and Marfan syndrome [33, 34]. Autosomal dominant polycystic kidney disease (ADPKD) is also associated with increased prevalence of diverticulosis, reaching $75 \%$ of ADPKD-affected patients [35]. The diverticula are predominantly right-sided, and severity of diverticulitis in these patients is markedly increased.

In conclusion, this study confirmed that diverticulosis is a significant problem in our country, especially for elderly patients. Further studies are warranted to reveal more about the pathogenesis 
and risk factors to enable primary prevention. The genetic component to diverticulosis will certainly require more research, as identification of responsible genes and, consequently, patients at risk might influence the necessary lifestyle changes or treatment options, preventing progression of the disease. To test the hypothesis that diverticulosis is associated with genetic polymorphism predisposing to protrusions in vulnerable connective tissue, an association study is planned, comparing the frequencies of genetic variants between the cases of diverticulosis and control patients.

In conclusion, in the studied population diverticula predominantly occurred in the sigmoid and descending colon. Their prevalence and distribution were similar to other Caucasian populations but higher than described in the earlier Polish study.

\section{Acknowledgments}

It is a pleasure to express our gratitude to Prof. Jacek Muszyński, Department of Gastroenterology and Internal Medicine, for providing facilities to undertake this investigation, and Prof. Jarosław Reguła, National Cancer Center, for sharing the data from the Colorectal Cancer Screening Program database.

\section{Conflict of interest}

The authors declare no conflict of interest.

\section{References}

1. Etzioni DA, Mack TM, Beart RW Jr, Kaiser AM. Diverticulitis in the United States: 1998-2005: changing patterns of disease and treatment. Ann Surg 2009; 249: 210-7.

2. West AB. The pathology of diverticulosis: classical concepts and mucosal changes in diverticula. J Clin Gastroenterol 2006; 40: 126-131.

3. Shahedi K, Fuller G, Bolus R, et al. Long-term risk of acute diverticulitis among patients with incidental diverticulosis found during colonoscopy. Clin Gastroenterol Hepatol 2013; 11: 1609-13.

4. Lewis LM, Banet GA, Blanda M, Hustey FM, Meldon SW, Gerson LW. Etiology and clinical course of abdominal pain in senior patients: a prospective, multicenter study. J Gerontol A Biol Sci Med Sci 2005; 60: 1071-6.

5. Reddy VB, Longo WE. The burden of diverticular disease on patients and healthcare systems. Gastroenterol Hepatol 2013; 9: 21-7.

6. Burkitt D. Diverticular disease of the colon epidemiological evidence relating it to fibre-depleted diets. Trans Med Soc Lond 1973; 89: 81-4.

7. Floch MH, Bina I. The natural history of diverticulitis: fact and theory. J Clin Gastroenterol 2004; 38: 2-7.

8. Peery AF, Sandler RS, Ahnen DJ, et al. Constipation and a low-fiber diet are not associated with diverticulosis. Clin Gastroenterol Hepatol 2013; 11: 1622-7.

9. Ünlü C, Daniels L, Vrouenraets BC, Boermeester MA. A systematic review of high-fibre dietary therapy in diverticular disease. Int J Colorectal Dis 2012; 27: 419-27.
10. Burgell RE, Muir JG, Gibson PR. Pathogenesis of colonic diverticulosis: repainting the picture. Clin Gastroenterol Hepatol 2013; 11: 1628-30.

11. Wess L, Eastwood MA, Wess TJ, Busuttil A, Miller A. Cross linking of collagen is increased in colonic diverticulosis. Gut 1995; 37: 91-4.

12. Touzios JG, Dozois EJ. Diverticulosis and acute diverticulitis. Gastroenterol Clin N Am 2009; 38: 513-25.

13. Reichert MC, Lammert $F$. The genetic epidemiology of diverticulosis and diverticular disease: emerging evidence. United Eur Gastroenterol J 2015; 3: 409-18.

14. Song JH, Kim YS, Lee JH, et al. Clinical characteristics of colonic diverticulosis in Korea: a prospective study. Korean J Intern Med 2010; 25: 140-6.

15. Tănase I, Păun S, Stoica B, Negoi I, Gaspar B, Beuran M. Epidemiology of the diverticular disease - systematic review of the literature. Chirurgia (Bucur) 2015; 110: 9-14.

16. Blachut K, Paradowski L, Garcarek J. Prevalence and distribution of the colonic diverticulosis. Review of 417 cases from Lower Silesia in Poland. Rom J Gastroenterol 2004; 13: 281-5.

17. Loffeld RJ, Van Der Putten AB. Diverticular disease of the colon and concomitant abnormalities in patients undergoing endoscopic evaluation of the large bowel. Colorectal Dis 2002; 4: 189-92.

18. Everhart JE, Ruhl CE. Burden of digestive diseases in the United States part II: lower gastrointestinal diseases. Gastroenterology 2009; 3: 741-54.

19. Kang JY, Dhar A, Pollok R, et al. Diverticular disease of the colon: ethnic differences in frequency. Aliment Pharmacol Ther 2004; 19: 765-9.

20. Lin OS, Soon MS, Wu SS, Chen YY, Hwang KL, Triadafilopoulos $\mathrm{G}$. Dietary habits and right-sided colonic diverticulosis. Dis Colon Rectum 2000; 43: 1412-8.

21. Manousos O, Day NE, Tzonou A, et al. Diet and other factors in the aetiology of diverticulosis: an epidemiological study in Greece. Gut 1985; 26: 544-9.

22. Gear JS, Ware A, Fursdon P, et al. Symptomless diverticular disease and intake of dietary fibre. Lancet 1979; 1: 511-4.

23. Strate LL. Diverticulosis and dietary fiber: rethinking the relationship. Gastroenterology 2012; 142: 205-7.

24. Hodgson WJ. The placebo effect. Is it important in diverticular disease? Am J Gastroenterol 1977; 67: 157-62.

25. Brodribb AJ. Treatment of symptomatic diverticular disease with a high-fibre diet. Lancet 1977; 1: 664-6.

26. Ornstein MH, Littlewood ER, Baird IM, Fowler J, North WR, Cox AG. Are fibre supplements really necessary in diverticular disease of the colon? A controlled clinical trial. $\mathrm{Br}$ Med J (Clin Res Ed) 1981; 282: 1353-6.

27. Jun S, Stollman N. Epidemiology of diverticular disease. Best Pract Res Clin Gastroenterol 2002; 16: 529-42.

28. Warner E, Crighton EJ, Moineddin R, Mamdani M, Upshur R. Fourteen-year study of hospital admissions for diverticular disease in Ontario. Can J Gastroenterol 2007; 21: 97-9.

29. Annibale B, Lahner E, Maconi G, et al. Clinical features of symptomatic uncomplicated diverticular disease a multicenter Italian survey. Int J Colorectal Dis 2012; 27: 1151-9.

30. Granlund J, Svensson T, Olén O, et al. The genetic influence on diverticular disease - a twin study. Aliment Pharmacol Ther 2012; 35: 1103-7.

31. Strate LL, Erichsen R, Baron JA, et al. Heritability and familial aggregation of diverticular disease: a population-based study of twins and siblings. Gastroenterology 2013 ; 144: 736-42. 
32. Connelly TM, Bert AS, Hegarty JP, et al. The TNFSF15 gene single nucleotide polymorphism rs7848647 is associated with surgical diverticulitis. Ann Surg 2014; 259: 1132-7.

33. Pepin M, Schwarze U, Superti-Furga A, Byers PH. Clinical and genetic features of Ehlers-Danlos syndrome type IV, the vascular type. N Engl J Med 2000; 342: 673-80.

34. Eliashar R, Sichel JY, Biron A, Dano I. Multiple gastrointestinal complications in Marfan syndrome. Postgrad Med J 1998; 74: 495-7.

35. Pourfarziani V, Mousavi-Nayeeni SM, Ghaheri H, et al. The outcome of diverticulosis in kidney recipients with polycystic kidney disease. Transplant Proc 2007; 39: 1054-6. 